\title{
Acupuntura e Atenção Primária à Saúde: análise sobre necessidades de usuários e articulação da rede
}

\author{
Acupuncture and Primary Health Care: analysis \\ of users' needs and network integration \\ Acupuntura y Atención Primaria de Salud: análisis de las \\ necesidades de los usuarios e integración de la red \\ Daniela Dallegrave ${ }^{1 *}$, Camila Boff ${ }^{2}$, Juliano André Kreutz ${ }^{3}$
}

Palavras-chave: Acupuntura Atenção Primária à Saúde Terapias Complementares Sistema Único de Saúde

\footnotetext{
Keywords: Acupuncture Primary Health Care Complementary Therapies Unified Health System
}

\section{Resumo}

A inserção de práticas integrativas e complementares no Sistema Único de Saúde remonta à discussão do movimento da reforma sanitária brasileira. Desde a aprovação da Política Nacional de Práticas Integrativas e Complementares, em 2006, a oferta de acupuntura tem crescido nos serviços públicos de saúde como prática multidisciplinar. Os usuários que acessam o Serviço de Saúde Comunitária do Grupo Hospitalar Conceição (Porto Alegre, no Rio Grande do Sul) podem ser encaminhados para a realização de acupuntura por meio da central de marcação de consultas do município. 0 presente estudo teve como objetivo explorar os encaminhamentos para acupuntura realizados no Serviço de Saúde Comunitária. Trata-se de um estudo de caráter descritivo, com abordagem quantitativa. As fontes de coleta de dados foram os formulários de referência e contrarreferência dos encaminhamentos para acupuntura dentro do Serviço de Saúde Comunitária. Os resultados apontaram maior proporção de mulheres e pessoas com idade acima de 50 anos entre os pacientes referenciados. Observaram-se diferenças significativas nas medianas de 'dias de espera' para consulta entre as unidades de saúde vinculadas ao Serviço de Saúde Comunitária. Ainda, confirmou-se a hipótese inicial de que o principal motivo de encaminhamento para realização de acupuntura seria 'dor', inscrevendo-se na lógica ocidental de pensar a saúde e os diagnósticos.

\section{Abstract}

The introduction of integrative and complementary practices into Brazil's Unified Health System brings back the discussion on health reform in Brazil. After the approval of the National Policy on Integrative and Complementary Practices, in 2006, acupuncture has been more present in public health services as a tool for multidisciplinary practices. Grupo Hospitalar Conceição, in the North side of Porto Alegre, in the state of Rio Grande do Sul, has a Community Health Service, which makes use of the city's appointment center to offer its users acupuncture services. This study aimed at exploring the references for acupuncture practices in this environment. It was a descriptive study with quantitative approach. The data collection sources were the reference and counter-reference forms with referrals to acupuncture in the Community Health Service. The results showed that women and people over 50 years-old are more frequently referred to acupuncture; it also highlighted long wait lists, with significant differences in terms of length of the waiting among the health units. The study has also confirmed the initial hypothesis that pain is the main reason for referring patients to acupuncture, supporting the Western way of conceiving of health and diagnoses.

Serviço de Saúde Comunitária do Grupo Hospitalar Conceição; Programa de Pós-graduação em Educação da Universidade Federal do Rio Grande do Sul. danidallegrave@gmail.com

²Estratégia Saúde da Família de Florianópolis. milaboff@hotmail.com

${ }^{3}$ Escola de Saúde Pública do Rio Grande do Sul; Programa de Pós-graduação em Educação da Universidade Federal do Rio Grande do Sul. julianokreutz@gmail.com

${ }^{*}$ Autor correspondente.

Fonte de financiamento: nenhuma.

Conflito de interesses: declararam não haver.

Recebido em: 20/06/2011

Aprovado em: 19/12/2011 
Palabras clave:

Acupuntura

Atención Primaria de Salud Terapias Complementarias Sistema Único de Salud

\section{Resumen}

La inclusión de prácticas complementarias y de integración en el Sistema Único de Salud se remonta a la discusión del movimiento de reforma de salud en Brasil. Desde la aprobación de la Política Nacional de Prácticas Integrativas y Complementarias, en 2006, la acupuntura se ha expandido en los servicios de salud pública como una herramienta para la práctica multidisciplinaria. Los usuarios que acceden al Servicio de Salud de la Comunidad del Grupo Hospitalar Conceição (Porto Alegre, Rio Grande do Sul) pueden ser derivados a un centro de acupuntura a través de citas centrales en la ciudad. Este estudio tuvo como objetivo explorar las referencias a la especialidad de acupuntura realizadas en el Servicio de Salud de la Comunidad. Es un estudio descriptivo con un enfoque cuantitativo. Las fuentes de datos fueron los formularios de referencia y contrarreferencia de las derivaciones para acupuntura en el Servicio de Salud de la Comunidad. La encuesta mostró una mayor proporción de mujeres, así como los grupos de edad mayores de 50 años, y también largos tiempos máximos de espera, con diferencias significativas en la mediana de días de espera entre los servicios de salud. Sin embargo, se confirmó la hipótesis inicial de que la razón principal para la derivación a esta especialidad es el 'dolor', inscribiéndose en la lógica occidental de pensar acerca de la salud y de los diagnósticos.

\section{Introdução}

O Serviço de Saúde Comunitária (SSC) do Grupo Hospitalar Conceição é responsável pela Atenção Primária à Saúde (APS) de aproximadamente $108.000^{1}$ habitantes da cidade de Porto Alegre, no Rio Grande do Sul. Trabalha nos moldes da Estratégia Saúde da Família, com profissionais graduados em Psicologia, Terapia Ocupacional, Enfermagem, Odontologia, Medicina, Farmácia, Nutrição e Serviço Social, grande parte deles especialistas em Saúde da Família e Comunidade. A APS de qualidade tem alto poder resolutivo, mas não prescinde dos serviços de referência ${ }^{2}$. Em Porto Alegre, aqueles que demandam atendimento de especialidades diversas, são agendados em outros serviços, por meio de uma central para agendamento de consultas que é gerenciada pela Secretaria Municipal de Saúde. São disponibilizadas cotas de consultas por especialidade para as unidades de saúde, as quais, por sua vez, gerenciam a prioridade das referências dos seus usuários.

\section{Acupuntura e medicina tradicional chinesa}

Medicina Tradicional Chinesa (MTC) é a denominação atual para um conjunto de práticas tradicionais em uso na China, desenvolvidas durante milhares de anos da sua história.

A MTC teve sua origem ao longo do Rio Amarelo. Durante a Idade da Pedra, já eram utilizadas agulhas feitas de lascas de pedra, gradualmente substituídas por outras de ossos e bambu até chegar àquelas feitas de metais ${ }^{3-5}$. A MTC passou por diversas modificações ao longo de várias dinastias chinesas e da história moderna. Seu ressurgimento, na China, deu-se após a fundação da República Popular da China, por meio de políticas governamentais para a massificação de seu uso, do ensino e do desenvolvimento de pesquisas em universidades e, em 1975, da criação de centros de formação para acupunturistas estrangeiros ${ }^{4-6}$.
Atualmente, são oito os principais métodos utilizados na MTC: fitoterapia; acupuntura; tui na (massagem); dietoterapia; moxabustão; auriculoterapia; ventosas e práticas corporais ${ }^{6}$.

Esse tipo de Medicina fundamenta-se numa estrutura filosófica, sistemática e abrangente, que utiliza linguagem simbólica e busca compreender as leis da natureza e sua inter-relação harmônica com o homem, visando à integralidade ${ }^{7}$.

A acupuntura é uma das técnicas utilizadas na MTC para amenizar desequilíbrios de energias corporais, que estão entrelaçadas com os fluxos de energia universais ${ }^{8}$. Por isso, para realizar um diagnóstico, o médico chinês leva em consideração os diferentes aspectos relacionados aos modos de viver e de funcionar da pessoa que procura por cuidados.

Neste sentido, há grandes diferenças nos atos de pensar o doente e a doença de maneira ocidental e oriental, as quais não necessariamente são utilizadas para doenças ocidentalmente conhecidas como orgânicas. Na prática de cuidado às pessoas, encontram-se "muitos médicos que adotam uma postura mental ocidental quando lidam com conceitos abstratos chineses ou tentam forçar conceitos ocidentais den-

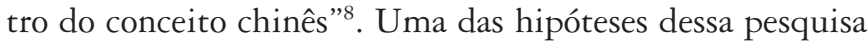
referia-se à adoção de tal 'postura mental ocidental', condicionando a maioria dos encaminhamentos realizados pelo serviço da Estratégia Saúde da Família.

No Ocidente, a prática mais conhecida e difundida da MTC é a Acupuntura. A difusão da Acupuntura está vinculada à companhia das índias ocidentais, em $1602^{3}$, e teve grande impulso com o trabalho do diplomata francês George Soulié de Morant, no século 20, que fez a tradução para o francês de importantes livros da MTC, disseminando os conhecimentos de acupuntura da França para a Europa e, posteriormente, às Américas ${ }^{3,9}$.

A Organização Mundial da Saúde (OMS) estimula, há décadas, o uso da acupuntura de forma integrada às técnicas da medicina moderna, por meio do reconhecimento de que "muitos elementos da medicina tradicional são bené- 
ficos, e a OMS incentiva e apoia os países para identificar soluções seguras, eficazes e práticas para uso em serviços públicos e privados de saúde"10. Em 1979, a MTC já reconhecia o uso da Acupuntura como terapêutica eficaz para mais de 40 doenças.

No Brasil, o desenvolvimento da acupuntura ocorreu de duas formas ${ }^{3,11}$. A primeira se iniciou pela chegada de imigrantes asiáticos no Sul e Sudeste, trazendo a Acupuntura como parte de sua bagagem cultural. Ao falar da história da cultura oriental, Shoji ${ }^{12}$ comenta que "os chineses são os mais antigos imigrantes do Extremo Oriente no Brasil, [...] é certo que algumas centenas de chineses desembarcaram no Rio de Janeiro em 1810, inicialmente trazidos para o cultivo de chá". Atualmente, o número de chineses e descendentes, no Brasil, é estimado em cerca de 190.000, dos quais 120.000 estão no Estado de São Paulo ${ }^{13}$.

Em 1895, firmou-se o Tratado de Amizade, Comércio e Navegação entre Brasil e Japão, iniciando-se o primeiro ciclo de imigração japonesa que vai de 1908 a 1925, com a chegada de 47.000 japoneses no país, levados, inicialmente, para as fazendas de café no estado de São Paulo. Grande parte desses imigrantes acabou se mudando para o interior paulista, para a região litorânea e para a periferia da capital. No segundo ciclo de imigração japonesa, de 1925 até 1941, chegaram mais 145.000 japoneses. Por fim, o terceiro compreende o grupo de imigrantes que chegaram após a Segunda Guerra Mundial. O Instituto Brasileiro de Geografia e Estatística (IBGE) constata que, até 1939, chegaram ao Brasil 185.799 imigrantes japoneses ${ }^{14}$.

A Imigração Coreana, no Brasil, teve início em 1963 quando estes chegaram ao Porto de Santos na condição de colonos agrícolas. Essa comunidade cresceu ao longo das décadas seguintes com a chegada de imigrantes legais, organizados pelo governo coreano, e de imigrantes ilegais, que chegaram ao Brasil pela fronteira dos países vizinhos, totalizando, hoje, cerca de 50.000 pessoas $^{12}$. Eles trouxeram na bagagem um tipo de acupuntura bastante diferenciado da chinesa e da japonesa. Na primeira metade do século 20, grande parte da acupuntura praticada por Orientais ficou restrita às suas comunidades, devido a dificuldades com a língua ${ }^{3}$.

A outra vertente se inicia com a vinda do professor Friedrich Johann Spaeth, que imigrou para o Brasil nos anos 1940, fugindo da violência nazista. Natural de Luxemburgo, fisioterapeuta e massoterapeuta, havia estudado Acupuntura na Alemanha. Em pouco tempo, ganhou grande clientela e chamou a atenção da comunidade científica-médica para a Acupuntura, formando o primeiro grupo organizado no país: a Sociedade Brasileira de Acupuntura e Medicina Oriental, em 1958 3 ,11.
A partir da década de 1980, a regulamentação da prática de acupuntura foi amplamente discutida, sendo alvo de disputa entre os conselhos profissionais da saúde. Atualmente, é reconhecida como especialidade pelos conselhos de Enfermagem, Farmácia, Fisioterapia e Terapia Ocupacional e Medicina. Os conselhos de Biomedicina, Fonoaudiologia e Psicologia têm regulamentações que habilitam os profissionais dessas áreas a utilizar a Acupuntura como recurso complementar ${ }^{15}$.

No Brasil, a Acupuntura vem se desenvolvendo nos últimos anos, sendo disponibilizada, na maioria das vezes, como serviço privado ou na saúde suplementar. Na saúde suplementar, pode-se observar um grande número de convênios que credenciam somente profissionais acupunturistas com formação em Medicina, o que faz pensar na manutenção da lógica hegemônica de categorização da doença pelo modo ocidental de pensá-la. Considera-se que, embora exista o reconhecimento da prática de outras profissões da saúde, isto não implica diferentes racionalidades, uma vez que o recorte biomédico é hegemônico no setor.

$\mathrm{Na}$ lógica da integralidade e da equidade, o Sistema Único de Saúde (SUS), público, gratuito e universal, de "direito de todos e dever do Estado"16 e que tem por diretriz a equidade, instituiu a Portaria $\mathrm{n}^{\circ}$ 971, referindo-se às Práticas Integrativas e Complementares, publicada em 2006, a qual trata a Acupuntura como uma técnica a ser oferecida nos serviços de saúde do SUS ${ }^{17}$. A regulamentação desta Portaria ocorre pela Política Nacional de Práticas Integrativas e Complementares (PNPIC), que sustenta que o uso da Acupuntura pode ser feito em casos de "promoção, manutenção e recuperação da saúde" ".

Na PNPIC, pode-se observar a ampliação das indicações de Acupuntura, quando esta refere que "admite-se, atualmente, que a estimulação de pontos de Acupuntura provoque a liberação, no sistema nervoso central, de neurotransmissores" 17 .

Essa concepção ampliada dos usos da Acupuntura aproxima-a do campo da APS, entendimento compartilhado por Kurebayashi" ${ }^{11}$, ao afirmar que "como a Acupuntura é indicada como parte de cuidados primários, seria de se esperar que os médicos de Atenção Primária tivessem interesse em praticá-la. Ela é utilizada por mais de $4 \%$ dos médicos de família do Reino Unido e é a segunda terapia mais popular, depois da Homeopatia".

$\mathrm{Na}$ sua prática profissional, os autores deste estudo observaram empiricamente que os encaminhamentos para Acupuntura são represados, na maioria das unidades de saúde do SSC, ou seja, haveria maior demanda do que oferta. Além disso, chamou a atenção, em observação não-sistemática no cotidiano do serviço, a frequência dos encaminhamentos para 
Acupuntura pelos mais diversos tipos e localizações de dor. Este fato levanta a hipótese de que há interferência na maneira como os profissionais realizam as referências para Acupuntura a partir do modo de pensar as doenças no Ocidente. Assim, este estudo teve como objetivo explorar os encaminhamentos para Acupuntura realizados no SSC do Grupo Hospitalar Conceição, no município de Porto Alegre, por meio da descrição do perfil dos usuários encaminhados e do levantamento dos motivos de encaminhamento.

\section{Métodos}

\section{Tipo de estudo}

Trata-se de uma pesquisa do tipo descritivo, com abordagem quantitativa. Os estudos descritivos são caracterizados pelo registro e descrição dos fatos observados, sem que o pesquisador interfira neles. O método descritivo é bastante utilizado nas pesquisas sociais para que se possa compreender melhor o fato pesquisado, pois proporciona uma nova visão do problema ${ }^{18}$.

Vale lembrar que os estudos do tipo descritivo não se propõem a conclusões, mas possibilitam o levantamento de diversas outras hipóteses.

\section{Local do estudo}

Constituíram local do estudo as 12 unidades de saúde do SSC do Grupo Hospitalar Conceição. A população atendida pelo SSC é proveniente dos mais diversos níveis culturais e socioeconômicos. Por ser um serviço com atendimento de Atenção Primária, constitui-se a principal porta do sistema de saúde e, portanto, pessoas com diferentes motivos de consulta são atendidas.

\section{Coleta de dados}

Esta foi uma pesquisa documental, em que os dados foram coletados a partir da ficha de referência e contrarreferência descrita como Acupuntura no campo especialidade e que estavam aguardando agendamento pela central de marcação da Secretaria Municipal de Saúde. Os dados foram digitados em banco de dados próprio, que foi criado no programa Assistente de Banco de Dados do BROffice.org.

As pesquisas documentais, para Prodanov e Freitas ${ }^{18}$, baseiam-se "em materiais que não receberam ainda um tratamento analítico".
A coleta de dados foi realizada em maio de 2010, e foram considerados os encaminhamentos datados até abril de 2010.

\section{Análise dos dados}

Os dados foram analisados segundo variáveis de tempo, lugar e pessoa.

De acordo com Rouquayrol e Almeida Filho ${ }^{19}$, variável é a "propriedade que determina a maneira pela qual os elementos de qualquer conjunto são diferentes entre si".

Foram considerados os seguintes itens:

- para a variável tempo - tempo de espera (definido pelo tempo máximo e mediana); distribuição da frequência dos encaminhamentos por mês, de todo o território do SSC;

- para a variável lugar - proporção de encaminhamentos por unidade de saúde;

- para a variável pessoa - sexo; faixa etária; agravo ou condição de saúde que foi identificado como o motivo de encaminhamento.

\section{Aspectos éticos}

A pesquisa foi conduzida dentro dos padrões éticos exigidos pela Comissão Nacional de Ética em Pesquisa / Conselho Nacional de Saúde / Ministério da Saúde (CONEP/CNS/ MS). O projeto foi aprovado no Comitê de Ética do Grupo Hospitalar Conceição, sob o parecer n n 10-048 e não houve qualquer conflito de interesse.

\section{Resultados}

Os atendimentos de Acupuntura, em Porto Alegre, integram a rede de atendimentos especializados e são oferecidos no Serviço de Acupuntura do Centro de Saúde Modelo, da Secretaria Municipal de Saúde de Porto Alegre, com uma oferta mensal total de 250 consultas, conforme informações obtidas por telefone. Já o Hospital Cristo Redentor disponibiliza duas novas vagas por semana para a Central de Marcação de Consultas. No SSC, atualmente, não há oferta de consultas para Acupuntura, embora existam alguns profissionais com formação especializada. Por vezes, estes profissionais utilizam a Acupuntura como recurso de sua prática clínica, embora no Serviço não haja disponibilização de materiais para tal prática.

Fizeram parte da pesquisa 296 encaminhamentos para a especialidade de Acupuntura, arquivados nas unidades de saúde e em espera para agendamento, em maio de 2010. Desses, 
cinco não apresentavam a data do encaminhamento. Os demais se distribuem do seguinte modo, por ano de referência: 2000: 1; 2002: 4; 2003, 12; 2004, 13; 2005, 31; 2006, 34; 2007, 74; 2008, 46; 2009, 55; 2010 (parcial), 21.

Nos documentos analisados, observa-se que a distribuição é diferente nas unidades de saúde, conforme a Figura 1. Uma vez que não há informações, por serviço, sobre o número de marcações efetuadas na Central da Secretaria $\mathrm{Mu}$ nicipal de Saúde, no período em questão, não é possível explicar essas diferenças. Neste estudo, não há como definir se tratam-se de diferenças nas frequências de encaminhamento ou da agilidade de marcação.

Destaca-se que, na Unidade de Saúde A, há o menor número de encaminhamentos, sendo dois $(66,67 \%)$ realizados em 2010. Na Unidade C, os encaminhamentos que aguardavam marcação eram de 2010. Na Unidade L, havia o maior número, sendo que dos 58 , apenas $4(6,9 \%)$ eram datados no ano da pesquisa.

Chama atenção o grande número de encaminhamentos datados de 2007 (74). Era de se esperar que a distribuição fosse homogênea, ao longo dos anos, ou que houvesse um crescimento na mesma proporção, nos anos mais próximos da atualidade, visto que o critério de agendamento é, na maioria das Unidades, a data mais antiga. Em 2000, tem-se 1 encaminhamento; em 2002, 4; em 2003, 12; em 2004, 13; em 2005, 31; em 2006, 34; em 2008, 46; em 2009, 55; e em 2010 (até maio), 21. Este fenômeno necessita de estudo específico para ser explicado. Os autores consideram que uma hipótese explicativa pode ser o lançamento da PNPIC em 2006 e seus desdobramentos.

Cabe destacar que não foi possível avaliar o tempo mínimo de espera, visto que seria necessária a investigação da frequência de marcação de consultas, na Central de Marcação das diferentes gerências distritais onde as unidades estão localizadas.

Figura 1. Encaminhamentos para Acupuntura, Serviço de Saúde Comunitária do Grupo Hospitalar Conceição, Porto Alegre, no Rio Grande do Sul, em espera para agendamento em maio de 2010.

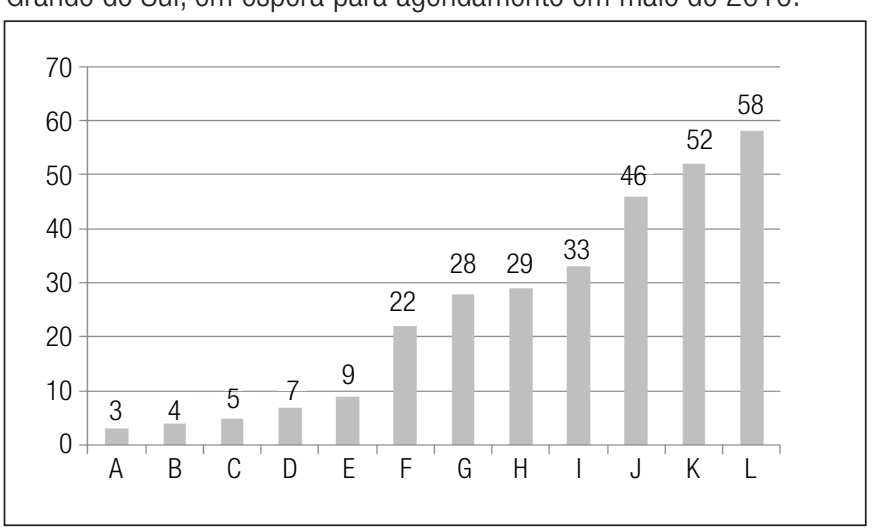

Com relação ao tempo máximo de espera dos encaminhamentos para a especialidade de Acupuntura que aguardam marcação, observa-se que, na Unidade I, o encaminhamento mais antigo data de 2000. No entanto, observa-se que esta não é a unidade com maior número de encaminhamentos, o que pode significar dificuldade de realizar o agendamento para moradores desta área (Tabela 1).

Avaliando a mediana de dias de espera dos encaminhamentos que aguardavam agendamento, na Figura 2, a Unidade F apresenta o maior tempo (1.764 dias). Cabe ressaltar que, durante a coleta de dados, constatou-se que essa unidade não organiza os encaminhamentos por ordem de data, o que foi observado nas demais unidades. Esse fato sugere que a Unidade organiza a demanda conforme outras prioridades que não a de tempo de encaminhamento, o que pode demonstrar equidade ou escolha aleatória.

Tabela 1. Distribuição dos encaminhamentos para Acupuntura, SSC/GHC, Porto Alegre, RS - em espera para agendamento em maio de 2010.

\begin{tabular}{lccc}
\hline Unidade & Encaminhamentos & Máximo Espera & Mediana Espera \\
\hline A & 3 & 260 & 53 \\
B & 4 & 497 & 114 \\
C & 5 & 120 & 119 \\
D & 7 & 1338 & 503 \\
E & 9 & 1482 & 905 \\
F & 22 & 2701 & 1764 \\
G & 28 & 1670 & 960 \\
H & 29 & 1792 & 1324 \\
I & 33 & 3645 & 857 \\
J & 46 & 2174 & 626 \\
K & 52 & 2514 & 970 \\
L & 58 & 2724 & 954 \\
Total & 296 & &
\end{tabular}

Figura 2. Mediana de dias de espera, por Unidade de Saúde, de Encaminhamentos para Acupuntura, SSC/GHC, Porto Alegre, RS, maio de 2010.

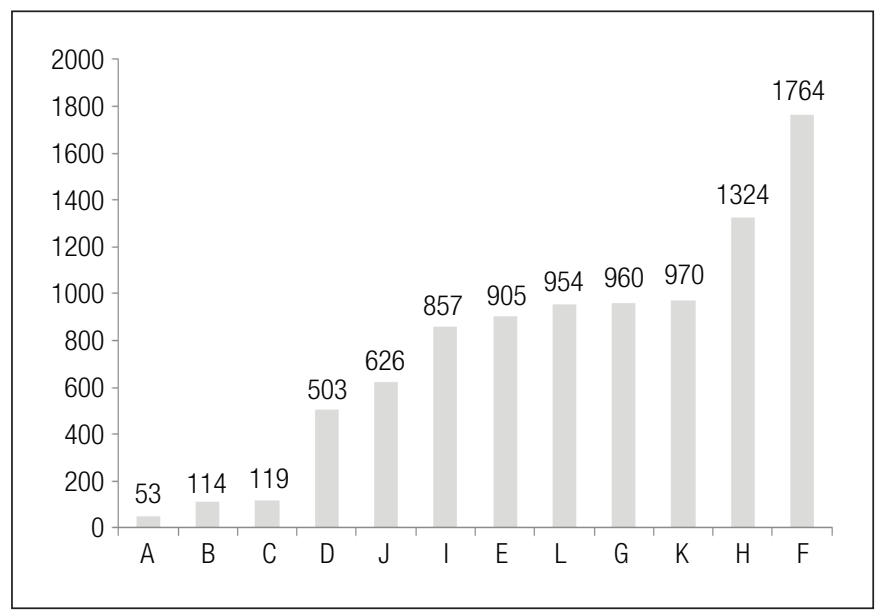


Dos encaminhamentos aguardando marcação no SSC do Grupo Hospitalar Conceição, 262 (88,5\%) eram de usuários do sexo feminino, o que pode demonstrar a predominância de acesso aos serviços de saúde pelas mulheres, conforme já descrito em diversos estudos ${ }^{20}$. Ainda, pode-se pensar que as queixas das mulheres enquadram-se melhor no que os profissionais consideram que sejam motivos tratáveis por Acupuntura.

Analisando a variável faixa etária, há predominância de encaminhamentos de usuários com idade entre 50 a 79 anos, perfazendo um total de 226 (76,3\%). Abaixo de 40 anos, apenas seis $(2,03 \%)$ encaminhamentos foram realizados. Assim, pode-se pensar em dois motivos: o primeiro que a população que menos acessa os serviços de saúde são os adultos jovens (abaixo de 40 anos), principalmente por estarem em horário de trabalho no mesmo tempo em que as unidades estão abertas; a segunda é que a principal razão de encaminhamentos para Acupuntura foi dor, perfazendo um total de 279 (94,3\%), e que este motivo, geralmente, não se caracteriza como acontecimento crônico nos jovens e eles costumam acessar as unidades por motivos agudos, o que está diretamente relacionado ao primeiro motivo. Essas hipóteses exigem investigação específica (Tabela 2).

Com relação aos médicos que encaminharam, percebe-se 182 $(61,5 \%)$ realizados por Médicos de Família e Comunidade. Houve pequena quantidade de interconsulta entre médicos de diferentes especialidades, observando-se que isso aconteceu quando o usuário foi encaminhado pela Central de Marcações para outra especialidade como traumato-ortopedista, por exemplo, e retornou a contrarreferência com a sugestão de consultar com acupunturista. Esse fato pode demonstrar a dificuldade de fluxo, na rede de serviços de saúde, os quais necessariamente são regulados pela Atenção Primária. Esta regulação evita usos inadequados do sistema de saúde, mas também pode causar prejuízos aos usuários que acabam ficando num jogo de vai-volta.

Dos encaminhamentos analisados, 12 (4,05\%) informavam que o usuário já havia realizado sessões de acupuntura.

Tabela 2. Distribuição dos encaminhamentos para Acupuntura, SSC - GHC, Porto Alegre, RS, em espera para agendamento em maio de 2010, por faixa etária e sexo.

\begin{tabular}{lccc}
\hline Faixa Etária & Encaminhamentos & Feminino & Masculino \\
\hline 10 a 19 anos & 1 & 0 & 1 \\
20 a 29 anos & 2 & 1 & 1 \\
30 a 39 anos & 3 & 2 & 1 \\
40 a 49 anos & 37 & 29 & 8 \\
\hline 50 a 59 anos & 91 & 84 & 7 \\
60 a 69 anos & 75 & 69 & 6 \\
70 a 79 anos & 60 & 55 & 5 \\
80 anos e mais & 21 & 17 & 4 \\
Ignorada & 6 & 5 & 1 \\
Total & 296 & 262 & 34 \\
\hline
\end{tabular}

Chama atenção que os usuários sobre os quais havia informação de acupuntura prévia eram do sexo feminino.

Analisando o motivo dos encaminhamentos para a especialidade de Acupuntura, observou-se que em 279 (94,26\%) foi por dor, o que confirmou a impressão e observação inicial dos pesquisadores.

Com relação à faixa etária, conforme a Figura 3, a proporção em que o motivo do encaminhamento para acupuntura foi de dor é de $100 \%$ dos usuários de 20 a 39 anos.

Quando analisou-se por sexo, foi possível observar uma prevalência de dor em 94,66\% dos encaminhamentos de mulheres. Já dos encaminhamentos para os homens, $91,18 \%$ foi por dor.

Considerou-se dor descrita a especificação da palavra 'dor' no campo 'motivo do encaminhamento'. Para dor subentendida, considerou-se a descrição de patologias, tais como doença degenerativa, artrite, artrose, hérnia de disco, fratura ou prótese e lesão por esforço repetitivo (LER/DORT).

Há que se considerar que, boa parte dessas dores crônicas pode estar relacionada a atividades laborais, o que pode ser motivo de outro estudo.

Observou-se que $14 \%$ dos encaminhamentos, nos quais havia dor descrita ou subentendida, também tinham menção a outra patologia, por exemplo, úlcera gástrica ou hipertensão arterial sistêmica, as quais geralmente estão relacionadas à restrição de tratamentos convencionais, ou seja, uso de medicamentos alopáticos.

Com relação à localização da dor descrita ou subentendida, pode-se observar um significativo número de encaminhamentos por dor em coluna cervical, torácica, lombar ou sacra, totalizando 118. Segue-se dor em membros inferiores com 70 encaminhamentos, conforme Figura 4.

Figura 3. Proporção de dor descrita ou subentendida, por faixa etária dos Encaminhamentos para Acupuntura, SSC/GHC, Porto Alegre, RS, em espera para agendamento em maio de 2010.

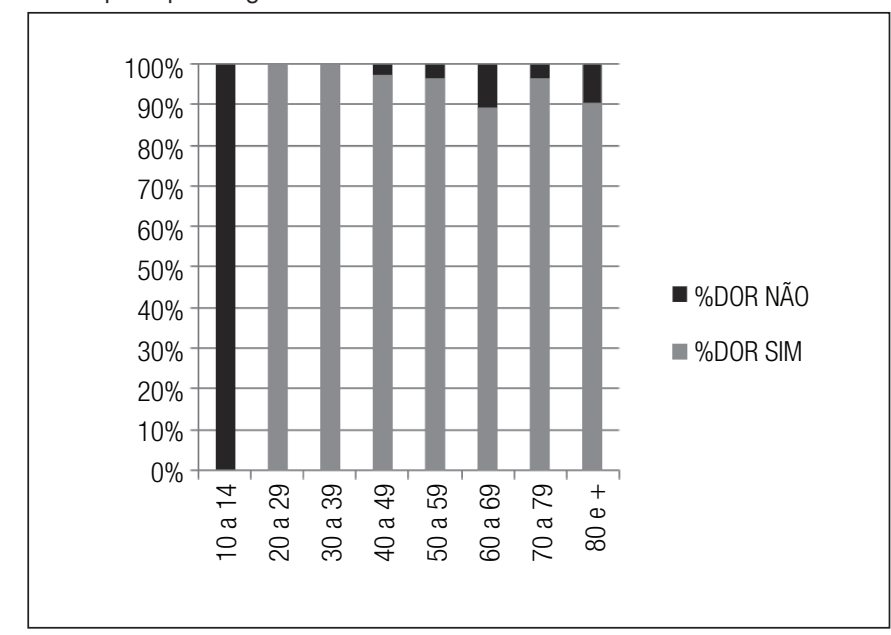


Alguns usuários encaminhados para Acupuntura apresentaram localização de dor em mais de um local, sendo consideradas tantas localizações quantas mencionadas, até o número de três regiões corporais. Quando era em mais de três regiões corporais, ou a descrição de fibromialgia, considerou-se como "poli", perfazendo 51 encaminhamentos.

Dor em ombro totalizou 23 encaminhamentos; membros superiores, 21; cabeça, 10; tórax, 5; face, 3; abdômen, 2 e quadril, 1 encaminhamento.

Relacionando a localização de dor por sexo, observa-se que $50(98,03 \%)$ dos encaminhamentos com descrição de mais de três localizações de dor foram de mulheres. Ainda, nas mulheres, distribui-se do seguinte modo a dor referida ou subentendida: $39 \%$ na coluna; $22 \%$ nos membros inferiores; $18 \%$ em mais de três localizações (poli); $8 \%$ nos ombros e $7 \%$ nos membros superiores. As demais, de menor proporção, referem-se à cabeça, ao tórax, abdômen e quadril. Por sua vez, nos homens, distribui-se assim: $39 \%$ na coluna; $29 \%$ nos membros inferiores; $16 \%$ na cabeça e $7 \%$ nos ombros. As demais referem-se à localização múltipla, aos membros superiores e ao tórax.

A localização de dor na coluna está predominante entre 50 e 79 anos, conforme a Tabela 3.

Figura 4. Localização da dor descrita nos encaminhamentos para acupuntura, SSC/GHC, Porto Alegre, RS, espera para agendamento, maio de 2010

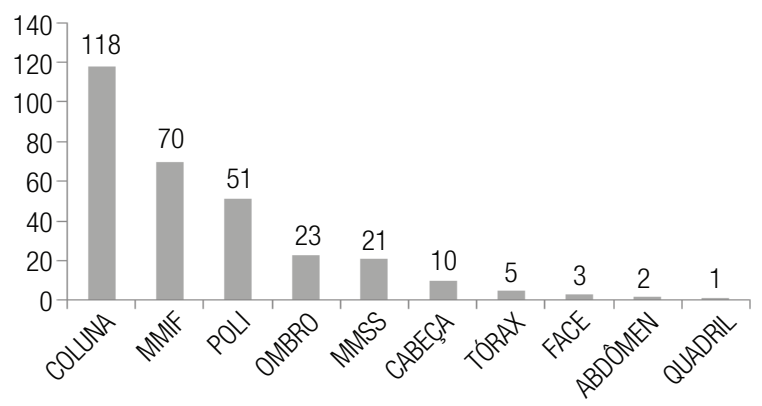

Tabela 3. Distribuição por Localização da Dor por Faixa Etária dos Encaminhamentos para Acupuntura, SSC/GHC, Porto Alegre, RS, em espera para agendamento em maio de 2010.

\begin{tabular}{lcccccc}
\hline Faixa etária & Coluna & MMSS & MMIF & Ombro & Poli & Outra \\
\hline 20 a 29 & 1 & 0 & 0 & 1 & 0 & 0 \\
30 a 39 & 3 & 0 & 2 & 0 & 0 & 0 \\
40 a 49 & 15 & 5 & 5 & 2 & 3 & 5 \\
\hline 50 a 59 & 34 & 7 & 23 & 5 & 17 & 10 \\
60 a 69 & 25 & 5 & 12 & 8 & 16 & 9 \\
70 a 79 & 29 & 1 & 23 & 5 & 8 & 3 \\
\hline 0 e + & 8 & 2 & 5 & 2 & 5 & 0 \\
\hline
\end{tabular}

\section{Discussão}

Neste estudo, notou-se a predominância de encaminhamentos por dor, referindo tentativas prévias de tratamento convencionais sem grande sucesso. O reconhecimento da eficácia da Acupuntura, para o universo médico estudado, parece referir-se aos casos de dor em que a terapêutica ocidental mostrou-se pouco ou não-efetiva para proporcionar alívio e bem-estar aos usuários, não configurando a Acupuntura como primeira escolha de tratamento. Isso pode refletir o desejo de resolutividade do profissional, que utiliza inicialmente as ferramentas com que está mais habituado, o conhecimento prévio do profissional sobre a baixa oferta do serviço na rede, ou ainda, a escolha da Acupuntura, como uma saída - para o profissional - aos possíveis sentimentos de impotência, experimentados frente a usuários que não respondem aos tratamentos normalmente propostos. Também inferi-se a dificuldade no manejo e entendimento desses usuários, bem como a inexistência de equipes multiprofissionais ampliadas, com a participação de outras categorias profissionais como educadores físicos e fisioterapeutas, para abordagem de pacientes com dor crônica ou recorrente na APS.

Existem maneiras diferentes de pensar o paciente e a doença nas culturas ocidental e oriental. A rede de serviços de saúde, no Brasil, atende à lógica ocidental. Mas, com advento da portaria $971^{15}$, pretende integrar outras lógicas de promoção e prevenção de saúde. A lacuna entre estas culturas ainda se configura em uma inadequação na oferta e no modo de acesso aos serviços de Acupuntura. Além da baixa oferta do serviço de Acupuntura, a organização acontece na lógica do atendimento especializado e não-integrada ao cotidiano da Atenção Primária. A política dos Núcleos de Apoio à Saúde da Família (NASF) possibilita a ampliação da equipe multiprofissional para a Atenção Primária, abrindo portas para a contratação de profissionais que atuem no campo das Práticas Integrativas e Complementares como a Acupuntura. Estas atividades podem ser exercidas diretamente pelos profissionais contratados nas Unidades de Saúde da Família ou indiretamente, por meio do apoio matricial aos trabalhadores que já tenham esta formação, mas que por motivos diversos não a exerçam no seu trabalho cotidiano. Vale ressaltar que a Acupuntura não é uma prática exclusiva da categoria médica e que, portanto, tanto seu exercício, como a indicação não devem ficar restritas a uma única categoria, para que, de fato, seja possível responder às complexidades na atenção à saúde.

Outra possibilidade dar-se-ia caso a rede pudesse pensar em itinerários diferentes, traçados pelos usuários e facilitados pelos processos de trabalho vividos nos serviços, e que fosse possível configurar em uma demanda para a Acupuntura que se autorregulasse. 


\section{Conclusões}

A Acupuntura, assim como a Homeopatia, segue outras lógicas de configuração do quadro do sujeito usuário, que as distanciam bastante do encadeamento de sinais e sintomas que configuram os diagnósticos atualmente utilizados pelos profissionais de saúde com formação tradicional ocidental, a qual segue a configuração da Classificação Internacional de Doenças (CID-10).

A pesquisa apontou maior proporção de mulheres referenciadas para a especialidade de Acupuntura, bem como pessoas das faixas etárias acima de 50 anos; destacou tempos máximos de espera elevados, com significativas diferenças nas medianas de dias de espera entre as unidades de saúde. Ainda, também confirmou a hipótese inicial de que o principal motivo de encaminhamento para esta especialidade seria a "dor", inscrevendo-se na lógica ocidental de pensar a saúde e os diagnósticos.

Caso houvesse maior disponibilidade de oferta dos serviços de terapias integrativas, a imagem de múltiplas práticas seria qualificada por conexões em uma rede diversa, de múltiplas racionalidades. Por outro lado, acredita-se que acupunturistas com as mais diversas formações de base podem alargar a linearidade que constitui atualmente os itinerários de cuidado - produção de rede para além do setor saúde, que dispute sentidos e produza outros saberes, na tentativa de não reduzir o andar da vida às práticas das ciências médicas.

Este artigo foi composto com uma ideia de que o serviço precisa ser regulado para não haver usos desnecessários, o que na maioria das vezes acarreta em altos custos. Porém, alguns modos de pensar a saúde podem e devem ser regulados de outras formas, para que não sejam subutilizados. Neste sentido, aponta-se para a necessidade de ampliar o debate com os profissionais que prestam assistência na Atenção Primária sobre as práticas integrativas, objetivando qualificar os encaminhamentos, bem como a necessidade da gestão da rede de serviços de saúde pautar as práticas integrativas como atividade essencial para produção da integralidade do cuidado, a fim de que tempos de espera próximos a dez anos não sejam admitidos em qualquer especialidade.

\section{Agradecimentos}

Apoio que recebemos do Grupo Hospitalar Conceição (GHC) para a realização desta pesquisa.

\section{Referências}

1. Brasil. Ministério da Saúde. Grupo Hospitalar Conceição. Serviço de Saúde Comunitária. [Internet]. Porto Alegre; 2011. [acesso em 2011 Nov 15]. Disponível em: http://www.ghc.com.br/default. asp?idMenu=2\&idSubMenu=5

2. Starfield B. Atenção Primária: Equilíbrio entre necessidades de saúde, serviços e tecnologia. Brasília: UNESCO, Ministério da Saúde; 2002.

3. Scognamillo-Szabo MVR, Bechara GH. Acupuntura: histórico, bases teóricas e sua aplicação em Medicina Veterinária. Cienc Rural. 2010; 40(2): 491-500. http://dx.doi.org/10.1590/S0103-84782010005000004

4. Maike SRL, Fundamentos Essenciais da Acupuntura Chinesa. São Paulo: Ícone; 1995. p. 29-31.

5. Jianping L, Yanliang C, Renhua S. Chinese acupuncture and moxibustion: a practical English - Chinese Library of Traditional Chinese Medicine. São Paulo: Publishing House of Shanghai College of Traditional Chinese Medicine; 1988. p. 2-12

6. Prefeitura Municipal de São Paulo. Secretaria Municipal de Saúde. Caderno Temático da Medicina Tradicional Chinesa. São Paulo; 2002. [acesso em 2010 Fev 27]. Disponível em: http://ww2.prefeitura.sp.gov.br//arquivos/ secretarias/saude/areas_tematicas/0047/MTC_CadernoTematico.pdf

7. Brasil. Ministério da Saúde. Secretaria de Atenção à Saúde. Departamento de Atenção Básica. Política Nacional de Práticas Integrativas e Complementares no SUS. Brasília: Ministério da Saúde; 2006.

8. Ross J. Zang Fu: sistemas de órgãos e vísceras da medicina tradicional chinesa. São Paulo: Roca; 1994

9. Lutaif S. George Soulié de Morant e Sua Tradução Ocidental do Saber Médico Chinês. 2005. [dissertação]. Pontifícia Universidade Católica: Porto Alegre; 2005.

10. World Health Organization (WHO). Guidelines on Basic Training and Safety in Acupuncture. 1999. [acesso em 2010 Fev 28]. Disponível em: http://whqlibdoc.who.int/hq/1999/WHO_EDM_TRM_99.1.pdf

11. Kurebayashi LFS, Oguisso T, Campos PFS, Freitas GF. Acupuntura na enfermagem brasileira: uma história em construção. Rev Paulista Enferm. 2007; 26 (2):127-33.

12. Shoji R. Imigração Chinesa e Coreana. 2004. [acesso em 2010 Mar 6]. Disponível em: http://www.pucsp.br/rever/rv3_2004/p_shoji.pdf

13. Kang S, Razzouk D, Mari JJ, Shirakawa I. The mental health of Korean immigrants in São Paulo, Brazil. Cad Saúde Pública. 2009; 25(4): 819-26. http://dx.doi.org/10.1590/S0102-311X2009000400013

14. Gonçalves RB. O sincretismo de culturas sob a ótica da arquitetura vernácula do imigrante japonês na cidade de Registro, São Paulo. An Mus Paul. 2008; 16(1): 11-46. http://dx.doi.org/10.1590/S010147142008000100002

15. Conselho Regional de Farmácia do Estado de São Paulo. Mais uma conquista: Acupuntura é especialidade Farmacêutica. 2009. [acesso em 2010 Mar 10]. Disponível em: http://www.crfsp.org.br/joomla/index. php?option=com_content\&view=article\&id=1839:mais-uma-conquistaacupuntura-e-especialidade-farmaceutica-\&catid=40:noticias\&ltemid=60

16. Brasil. Senado Federal. Constituição da República Federativa do Brasil de 1988. Brasília; 1988

17. Brasil. Ministério da Saúde. Portaria n 971, de 3 de maio de 2006. Aprova a Política Nacional de Práticas Integrativas e Complementares (PNPIC) no Sistema Único de Saúde. Brasília; 2006. [acesso em 2010 Fev 28]. Disponível em: http://portal.saude.gov.br/portal/arquivos/pdf/ PNPIC.pdf

18. Prodanov CC, Freitas EC. Metodologia do Trabalho Científico: métodos e técnicas da pesquisa e do trabalho acadêmico. Novo Amburgo: Feevale; 2009. 288p.

19. Rouquayrol MZ, Almeida Filho N. Epidemiologia e Saúde. $5^{a}$ ed. Rio de Janeiro: Medsi; 1999.

20. Viacava F, Travassos C, Pinheiro R, Brito A. Gênero e Utilização de Serviços de Saúde no Brasil. [acesso em 2011 Set 21]. Disponível em: http://ris.bvsalud.org/finals/BRA-3020.pd 